\title{
Comparativa del avance en desarrollo en las telecomunicaciones entre Ecuador y Bolivia.
}

\section{Comparison of progress in development in telecommunications between}

\section{Ecuador and Bolivia.}

José Alberto Cumbicos Romero. ${ }^{1}$, Lenin Patricio Jiménez Jiménez. ${ }^{2}$, María Cecilia Luna Alvarado. ${ }^{3}$, Ángel Leonardo Valdivieso Caraguay. ${ }^{4} \&$ Lorena Isabel Barona López. ${ }^{5}$

Recibido: 08-03-2017 / Revisado: 08-05-2017 Aceptado: 13-06-2018/ Publicado: 01-07-2018

\begin{abstract}
.
DOI: https://doi.org/10.33262/cienciadigital.v2i3.134

The telecommunications sector is one of the main engines for a country's development. A high level of connectivity facilitates productive activities improving the quality of life of people around the world. In this article the status of telecommunications between Ecuador and Bolivia are analyzed. The study includes a review of the legal situation and progress of the telecommunications sector in each country. In addition, this work presents a comparison of the most relevant parameters such as rates, fixed lines, mobile, interconnection, among others.
\end{abstract}

Keywords: Normative, Interconnection, Telecommunications.

\section{Resumen.}

El sector de las telecomunicaciones es uno de los principales motores para el desarrollo de un país. Un alto nivel de conectividad facilita el desarrollo de actividades productivas mejorando la calidad de vida de las personas. En el presente

\footnotetext{
${ }^{1}$ Universidad Técnica Particular de Loja, Departamento de. Ciencias de la Computación y Electrónica, Loja, Ecuador, jacumbicos@utpl.edu.ec

${ }^{2}$ Universidad Técnica Particular de Loja, Departamento de Ciencias de la Computación y Electrónica, Loja, Ecuador, lpjimenez@utpl.edu.ec

${ }^{3}$ Universidad Técnica Particular de Loja, Departamento de Ciencias de la Computación y Electrónica, Loja, Ecuador,mcluna2@utpl.edu.ec

alvaldivieso@utpl.edu.ec.

${ }^{4}$ Universidad Técnica Particular de Loja, Departamento de Ciencias de la Computación y Electrónica, Loja, Ecuador, alvaldivieso@utpl.edu.ec.

${ }^{5}$ Escuela Politécnica Nacional, Facultad de Sistemas, Quito, Ecuador, lorena.barona@epm.edu.ec
} 
artículo se analiza la situación de las telecomunicaciones entre Ecuador y Bolivia. El estudio incluye un análisis de la situación legal y avance tecnológico del sector de las telecomunicaciones de cada país. Además, el trabajo presenta comparativas de los parámetros más relevantes tales como tarifas, líneas fijas, móviles, interconexión, entre otros.

Palabras Claves: Normativa, Interconexión, Telecomunicaciones.

\section{Introducción .}

Los servicios que hoy en día se crean a partir del despliegue de infraestructura de telecomunicaciones permiten llevar a cabo relaciones laborales y sociales que mejoran la calidad de vida de las personas. La dependencia y utilidad que se crea por diferentes servicios de telecomunicaciones mueve la economía de un país, por tanto se considera a este sector un motor que fomenta el desarrollo integral de un país.

Según un estudio llevado a cabo por el Banco Interamericano de Desarrollo (BID), un aumento del $10 \%$ en la penetración de servicios de banda ancha en América Latina y el Caribe (ALC) involucra un incremento promedio de 3,2\% del PIB, un aumento de la productividad del 2,6\% y crea 67,016 nuevos empleos (Zaballos \& López-Rivas, 2012). Por otra parte, (IBM, 2008) menciona que por cada empleo directo que se crea en el sector de las TIC, se generan otros seis empleos indirectos y según (IBM, 2017), la industria de las TIC en 2016 genero aproximadamente cien mil empleos a nivel global.

Desde una perspectiva regional, según datos de la Unión Internacional de Telecomunicaciones (UIT), la penetración de banda ancha fija y móvil en ALC es del 10\% y $30 \%$ respectivamente. La tasa de trasmisión media de las conexiones fijas en los países de ALC en 2016 fue de 4,64 Mbps, mientras que para conexiones móviles la media para ALC fue de 3,87 Mbps. El 27\% de la población de ALC está cubierta por redes de cuarta generación de banda ancha móvil (4G). En cuanto a los hogares con acceso a internet, el promedio de ALC es del 44\% (Cabrera \& Gabarr, 2017). Finalmente, un factor que condiciona directamente el uso de servicios de telecomunicaciones es el precio. Según (Zaballos \& Rodríguez, 2017), los ciudadanos de ALC tienen que emplear 10\% de su ingreso mensual tanto para banda ancha fija como para móvil.

En el presente artículo se realiza el análisis general del estado de las telecomunicaciones, en el ámbito legal y técnico, tanto de Ecuador como de Bolivia. En la sección 1 se presenta información relacionada a la infraestructura actual de telecomunicaciones y datos de operadores que se encuentran en Ecuador y Bolivia. En la sección 2 se detallan las características importantes de las respectivas leyes que rigen las telecomunicaciones en cada país. Finalmente, en la sección 3 se realiza una comparativa de datos entre Ecuador y Bolivia concernientes al sector de las telecomunicaciones. 


\section{Marco Técnico.}

En la siguiente sección se presentan las principales características del sector de las Telecomunicaciones con respecto al ámbito técnico.

\subsection{Ecuador.}

Según el Plan Nacional de Telecomunicaciones y Tecnologías de Información del Ecuador, publicado en el año 2016, los avances en el sector de telecomunicaciones y TICs han tenido un notable crecimiento. En base a la información provista por el Banco Central del Ecuador en el 2013, el sector de Correros y Telecomunicaciones representa el $2.1 \%$ del PIB en Ecuador (Ministerio de Telecomunicaciones y de la Sociedad de la Información, 2016).

En los últimos años ha aumentado la penetración de los servicios básicos de telecomunicaciones en el país. En el año 2015 la cobertura poblacional de servicios móviles fue del $96 \%$ en comparación a la cobertura existente en el año 2006 que era del $89 \%$. De la misma forma el desarrollo y el despliegue de la infraestructura de redes SMA 3G y 4G es significativamente mayor teniendo en el 2015 una cobertura poblacional del 90\% y 32\% respectivamente, a diferencia de la cobertura existente de la red 3G en el 2006 que era de aproximadamente el 53\%. Otro punto importante es el crecimiento del número de suscripciones de banda ancha fija en el periodo 2006-2015 gracias al despliegue de más de $60000 \mathrm{~km}$ de fibra óptica a nivel nacional (Ministerio de Telecomunicaciones y de la Sociedad de la Información, 2016).

Actualmente, en el Ecuador, existen 3 cables submarinos que manejan el tráfico internacional estos son: cable submarino Pan American (PAN-AM), South America (SAm1) y Pacific Caribbean Cable System (PCCS). PAN-AM al igual que SAm-1 aterrizan en Punta Carnero y tienen una capacidad de 190 Gbps y 1.92 Tbps respectivamente. PCCS aterriza en la ciudad de Manta y tiene una capacidad de 20 Tbps (Jaya, 2017; Montenegro \& Sánchez, 2016; TELECOMReview, 2015; Velasco, 2014; Wholesale Services, n.d.).

De acuerdo a (Zabala \& Adrade, 2016) las compañías de telecomunicaciones existentes en el Ecuador, conforme a la Clasificación Internacional Industrial Uniforme (CIIU), se clasifican en los siguientes segmentos (Tabla 1).

Tabla 1.Composición del Sector de las Telecomunicaciones por su Actividad en los años 2015 y 2016.

\begin{tabular}{lcc}
\hline \multicolumn{1}{c}{ Actividad } & Nro. empresas 2015 & Nro. empresas 2016 \\
\hline Venta de equipos de comunicación & 261 & 261 \\
Transmisión de imágenes, sonido, datos y otra información & 131 & 129 \\
Comunicaciones telefónicas & 19 & 19 \\
Venta de equipos de comunicaciones & - & 138 \\
\hline
\end{tabular}

Nota. En el año 2016 existe un mayor número de empresas. Fuente: (EKOS, 2017;

Zabala \& Adrade, 2016). 
En el año 2015 se reportó un ingreso de 4489.7 millones de dólares por parte de las empresas que conforman el sector de las telecomunicaciones. Se evidencia una concentración de mercado ya que el $70 \%$ de los ingresos totales se encuentran distribuidos entre 3 empresas: CONECEL S.A, CNT EP y OCTECEL S.A.(Zabala \& Adrade, 2016). Sin embargo, en el año 2016 el sector de las telecomunicaciones generó ingresos por 4072.9 millones de dólares lo que significó una tasa de variación anual mayor al menos $6 \%$ respecto al ingreso generado en el año 2015 (EKOS, 2017). En la Tabla 2, se presenta un reporte de los ingresos obtenidos por parte de las empresas de telecomunicaciones con concentración de mercado en el país.

Tabla 2.Ingresos de las Empresas de Telecomunicaciones con concentración de mercado en el periodo 2014-2016.

\begin{tabular}{lccc}
\hline Empresa & 2014 & 2015 & 2016 \\
\hline CONECEL S.A. & 1671.42 & 1540.23 & 1446.16 \\
CNT EP. & 757.80 & 994.63 & 934.56 \\
OCTECEL S.A. & 689.56 & 683.00 & 680.63
\end{tabular}

Nota. Los ingresos se muestran en USD millones. Fuente: (EKOS, 2017; Zabala \& Adrade, 2016)

Con el fin de tener una idea más clara sobre las tarifas que tienen estos operadores en la Tabla 3 se muestran los costos y detalles de algunos de los servicios básicos que ofrecen al mercado. Adicionalmente, es importante recalcar que CNT EP es la corporación propietaria de la red de fibra óptica más grande del país ya que cuenta con más de $13969 \mathrm{~km}$ de fibra óptica desplegada a nivel nacional (Zabala \& Adrade, 2016). Sin embargo, la mayoría de esta infraestructura se concentra en la Costa y Sierra.

Tabla 3.Tarifas de los servicios básicos de los operadores con poder de mercado en el ecuador en USD.

\begin{tabular}{lcccc}
\hline Operadora & & Claro & CNT & Movistar \\
\hline \multirow{2}{*}{ Telefonía Móvil } & Prepago & 2.00 & 4.48 & 3.00 \\
Telefonía Fija & Postpago & 16.80 & 11.20 & 15.00 \\
Internet & & 6.94 & 6.94 & - \\
TV & 22.39 & 23.41 & 24.99 \\
Triple Play & & 20.60 & 18.50 & - \\
\hline
\end{tabular}

Nota. Las tarifas entre operadores son similares. Fuente: (CLARO, 2018; CNT EP, 2018; Movistar, 2018). 
Según estadísticas publicadas por la ARCOTEL en Abril del 2018, actualmente el número total de líneas activas del servicio móvil avanzado es 14.893.704. De las cuales, CONECEL tiene el $53.72 \%$, le sigue OTECEL con el $30.32 \%$ y finalmente CNT con $15.87 \%$ líneas activas (ARCOTEL, 2018b). De acuerdo al índice de desarrollo TIC publicado por la ITU en 2017 el ancho de banda por usuario de internet en Ecuador es de 43.67 Kbps (ITU, 2017a). En cuanto al número de suscripciones al servicio de Internet en el Ecuador se tiene un total de 1.78 millones y 8.81 millones de usuarios suscritos a los servicios de banda ancha fija y móvil (ARCOTEL, 2018a).

\subsection{Bolivia.}

Durante el año 2016 el sector de las telecomunicaciones creció en $2.04 \%$ respecto al año anterior, siendo el menor incremento registrado en los últimos años. El aporte que representa el sector al PIB es del 5\% en el mismo año (ATT, 2017b). En la figura 1 se observa el crecimiento que han tenido las Telecomunicaciones.

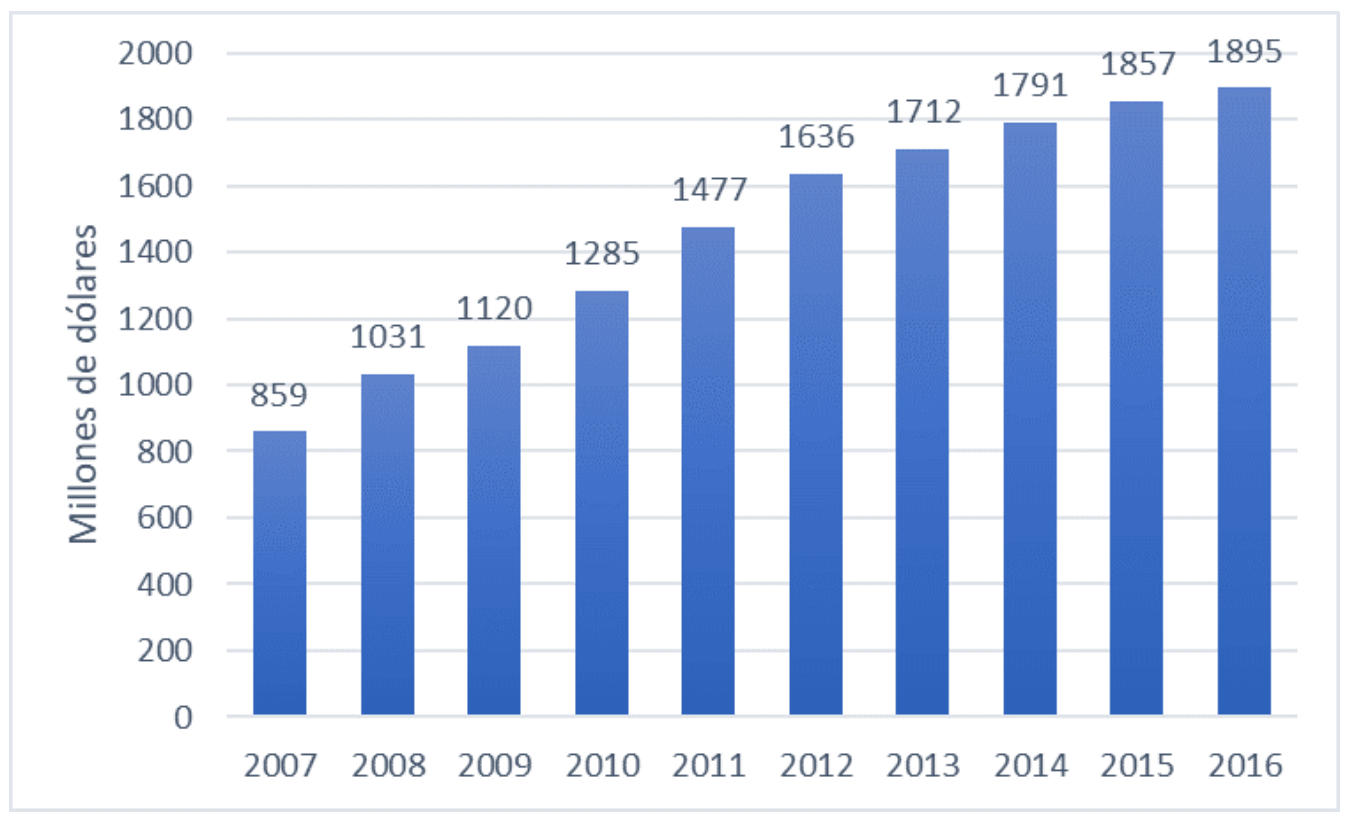

Figura 1. Evolución de Ingresos Netos del sector de Telecomunicaciones. Fuente: (ATT, 2017b).

A nivel nacional existen tres empresas que prestan el servicio móvil y son: ENTEL, NUEVATEL - VIVA y TELECEL- TIGO. La distribución del mercado es el siguiente: ENTEL tiene una participación del $44.91 \%$, le sigue TELECEL-TIGO con $30.11 \%$ y NUEVATEL-VIVA con el 24.98\% (ATT, 2016). En el 2017 se incrementaron cerca de 500 mil líneas móviles superando los dos años anteriores que tuvieron un estancamiento en el crecimiento (figura 2). La penetración del servicio móvil es del 94\% y a marzo de 2017 las líneas de modalidad prepago representan el $89 \%$ del total, mientras que el $11 \%$ restante corresponde a la modalidad postpago (ATT, 2017b). 


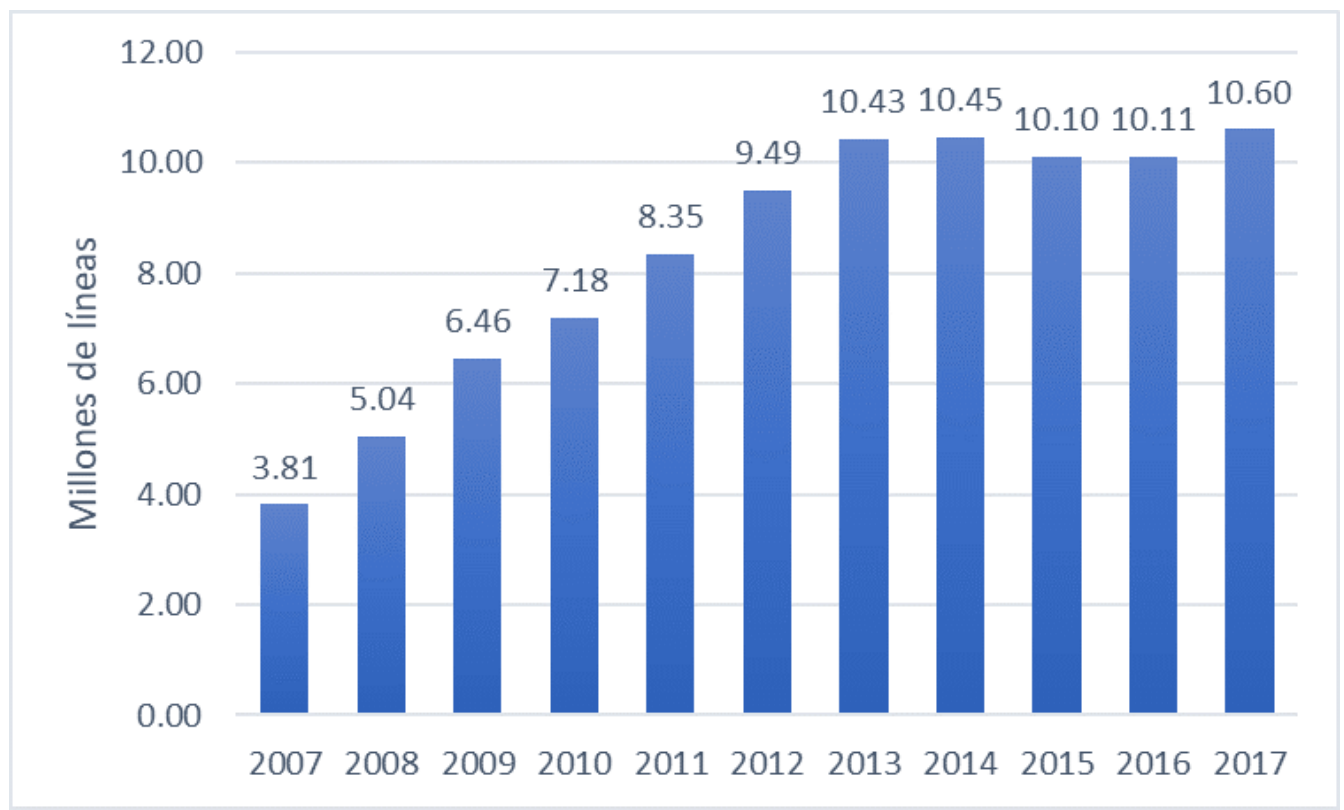

Figura 2. Líneas móviles registradas. Fuente: (ATT, 2017a).

En cuanto al número de conexiones a Internet, durante los últimos años ha existido un crecimiento exponencial (figura 3). La penetración del servicio de Internet es del 78.4\%. La tecnología FTTx tuvo un crecimiento de más de $122.6 \%$ y el mayor número de conexiones a Internet se realiza por medio de dispositivos móviles utilizando tecnologías de 2.5 a $4 \mathrm{G}$, representando un $88.79 \%$ del total. A junio de 2017 se cuenta con una conectividad Internacional de Internet de 195.132 Mbps (ATT, 2017b).

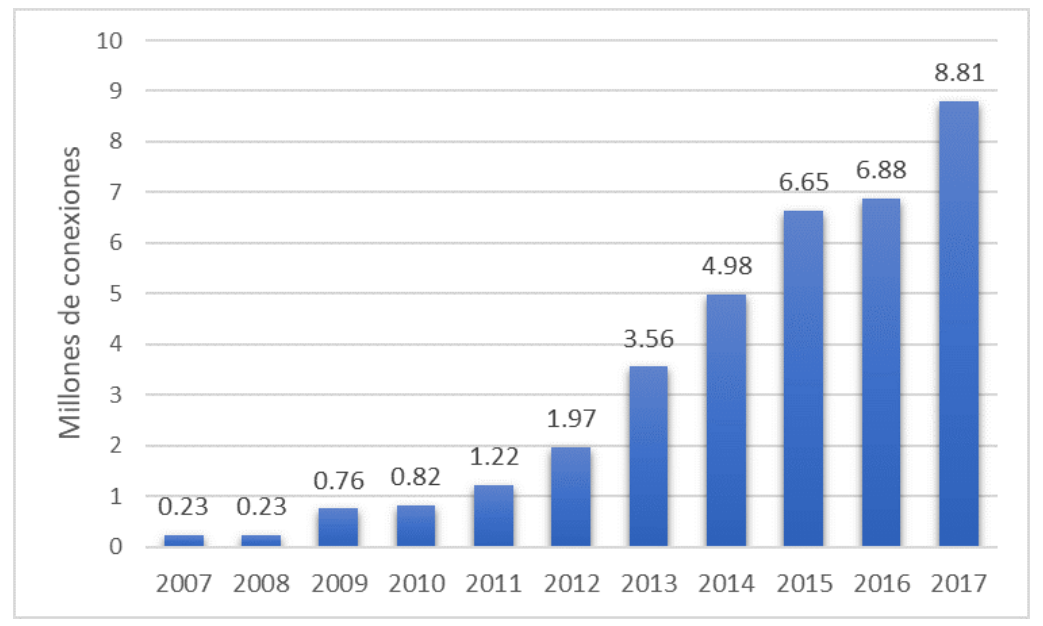

Figura 3. Número de conexiones a Internet. Fuente: (ATT, 2017a).

Bolivia se conecta al cable submarino Nautilus a través de dos ramas: la primera es 
haciendo tránsito por Buenos Aires y la segunda por Chile, siendo la segunda la más utilizada. Sin embargo, en ambos casos la red desciende a estas ciudades para luego subir a Miami, aumentando el tiempo de propagación con retardos de 100 milisegundos, en consecuencia, la latencia de la red aumenta. También se dispone de una baja capacidad de conexión con el cable Pan-Am a través de nodos ubicados en Chile o Argentina, lo que implica hacer tránsito por estos países ya que no se cuenta con una salida directa al océano (Campero, 2016).

El satélite geoestacionario Tupac Katari fue otra opción que implementó Bolivia para mejorar los servicios de Telecomunicaciones, fue lanzado en el 2013 y tuvo un costo de $\$ 300$ millones. Este se utiliza principalmente para llegar a zonas rurales que no tienen acceso a estos servicios y permitir el desarrollo de esta población (EABOLIVIA, 2013). Para zonas urbanas es muy deficiente por las desventajas propias del sistema tal como retardo adicional y un ancho de banda bajo. De acuerdo al índice de desarrollo de ICT publicado por la ITU en 2017 el ancho de banda por usuario de internet en Bolivia es de 36.34 Kbps (ITU, 2017a).

\section{Marco Legal.}

A continuación, se analizarán las normativas vigentes en cuanto al sector de las telecomunicaciones en los países de Ecuador y Bolivia.

\subsection{Ecuador.}

El sector de las telecomunicaciones en Ecuador es regido por la Ley Orgánica de Telecomunicaciones (LOT) elaborada en el año 2015 y la entidad reguladora de este sector es la Agencia de Regulación y Control de las Telecomunicaciones (ARCOTEL) (Cabrera \& Gabarr, 2017). Dentro de la LOT se establecen normativas para el uso del espectro radioeléctrico en el país (Asamblea Nacional, 2015), algunas de estas serán descritas a continuación.

En la LOT se establece que las redes públicas de telecomunicaciones deben tener un diseño de red abierto de modo que permita la interconexión, acceso y conexión (Asamblea Nacional, 2015). La manera en que está distribuido el espectro radioeléctrico es: 33\% para la operación de medios públicos, $33 \%$ para la operación de medios privados y finalmente $34 \%$ para la operación de medios comunitarios, siendo estos últimos priorizados en la distribución o asignación de frecuencias (Asamblea Nacional, 2013).

Los títulos habilitantes para la prestación de servicios de telecomunicaciones son: concesión, autorización y registro de servicios. El otorgamiento de los mismos se realiza mediante dos modalidades: adjudicación directa y proceso público competitivo. Por adjudicación directa se realiza cuando son frecuencias no esenciales o bandas de uso compartido. Por proceso público competitivo cuando el número de solicitantes supera la 
cantidad de frecuencias disponibles o cuando se destinen a la prestación de servicios de carácter masivo. Las concesiones y autorizaciones otorgadas por la ARCOTEL tienen una duración máxima de 15 años (Asamblea Nacional, 2015), exceptuando a los operadores de cable submarino y empresas públicas de telecomunicaciones, cuya concesión es de 20 años (ARCOTEL, 2016; Asamblea Nacional, 2013).

Los prestadores de servicios de telecomunicaciones podrán establecer libremente sus tarifas, esto siempre que no sobrepasen los techos tarifarios establecidos por la ARCOTEL. Con el fin de promover la competencia en la LOT se define un pago por concentración de mercado al operador preponderante, es decir al prestador de servicios de telecomunicaciones que tenga más del $50 \%$ de abonados, clientes suscriptores, líneas activas y tráfico en un determinado servicio. El operador con poder de mercado pagará al Estado un porcentaje de sus ingresos totales anuales como se muestra en la Tabla 4.

Tabla 4.Porcentaje de pago por concentración de mercado.

\begin{tabular}{ccc}
\hline Desde & Hasta & Pago \\
\hline $30 \%$ & $34.99 \%$ & $0.5 \%$ \\
$35 \%$ & $44.99 \%$ & $1 \%$ \\
$45 \%$ & $54.99 \%$ & $3 \%$ \\
$55 \%$ & $64.99 \%$ & $5 \%$ \\
$65 \%$ & $74.99 \%$ & $7 \%$ \\
$75 \%$ & En adelante & $9 \%$
\end{tabular}

Nota. El máximo porcentaje de pago por concentración de mercado es del 9\%. Fuente: (Asamblea Nacional, 2015)

En la LOT se establece como derecho de los abonados, clientes y usuarios la portabilidad numérica, La cual permite mantener su número telefónico cuando el abonado haya decidido cambiar de operadora (ARCOTEL, 2018a). En cuando a la interconexión, se establece que todos los prestadores de servicios tienen la obligación de interconectarse con otras redes públicas y permitir la oportuna interconexión a su red. Para esto, deberán poseer diseños de arquitectura de red abierta que permitan la interoperabilidad de sus redes (Asamblea Nacional, 2015).

La interconexión puede ser realizada en cualquier punto de la red siempre y cuando sea técnica y económicamente factible. El prestador de servicios de telecomunicaciones que solicite la interconexión es responsable de cubrir los gastos de inversión, operación y mantenimiento de las instalaciones utilizadas para llegar al punto de enlace con la otra red (CONATEL, 2007). Cuando no exista autorización previa por parte de la ARCOTEL no se puede procederse a la desconexión, interrupción, suspensión o cierre de la interconexión ya sea de forma unilateral o de mutuo acuerdo (Asamblea Nacional, 2015). 


\subsection{Bolivia.}

En el Estado Plurinacional de Bolivia se estima que existen 11285500 habitantes, según datos del INE (INE, 2018). Entre las disposiciones legales en el sector de las Telecomunicaciones se tiene la "Ley General de Telecomunicaciones y Tecnologías de Información y Comunicación” creada en el año 2011 (ADSIB, 2011) y el "Reglamento General a la Ley N 164 General de Telecomunicaciones y Tecnologías de Información y Comunicación, para el Sector de Telecomunicaciones" creada en el 2012 (ATT, 2012). La institución que hace cumplir el marco legal es la Autoridad de Regulación y Fiscalización de Telecomunicaciones y Transporte (ATT).

En la Ley General se establece la distribución de frecuencias de la siguiente forma: Estado el 33\% definidas por el Órgano Ejecutivo, comercial el 33\% asignadas por licitación pública, social comunitario el $17 \%$ y pueblos indígenas, comunidades interculturales y afro bolivianas el $17 \%$, asignadas por concurso de proyectos. También se indica que para proveer servicios y operar redes de telecomunicaciones se requiere una licencia única o de radiodifusión y suscribir un contrato con la entidad reguladora. Para el caso de las licencias, no serán otorgadas a personas naturales o jurídicas extranjeras. Se prohíbe otorgar este documento a los dignatarios y personas que tengan relación de parentesco hasta el primer grado de consanguinidad, de los órganos ejecutivo, legislativo, judicial, electoral.

Un tema a tener en cuenta en el ámbito legal en Bolivia es la portabilidad numérica. Hasta la fecha el país no cuenta con un reglamento que brinde este derecho a los abonados de las diferentes operadoras. Según (Consulado de Bolivia, 2017) el 9 de septiembre de 2016, la ATT tuvo una solicitud de los tres operadores (Entel, Telecel, Nuevatel), que pedían postergar la aplicación de la portabilidad, argumentando temas de carácter técnico y económico. Atendiendo ese pedido, el Gabinete Ministerial aprobó el Decreto Supremo 3004, que da un nuevo plazo para su aplicabilidad.

Para el caso de la interconexión, es obligatorio que las redes públicas estén interconectadas. La ATT podrá limitar la interconexión en caso de que las redes no sean técnicamente ni funcionalmente compatibles, representen un peligro a la red solicitada o amenace la vida o seguridad de los usuarios. La interrupción total o parcial debe hacerse con previa autorización del ente regulador (ADSIB, 2011). En el Reglamento General se señala que un proveedor tiene posición dominante cuando cumple con dos condiciones: tener el $40 \%$ de participación de los ingresos brutos en el mercado y que la diferencia con el segundo competidor sea del $10 \%$. Adicionalmente, estará sujeto a las tarifas del Régimen de Tope de Precios (ATT, 2012).

\section{Análisis Comparativo.}

De lo expuesto en las secciones 1 y 2 se realiza una comparación de los datos más relevantes en cuanto al sector de las telecomunicaciones como los son: aporte al PIB de cada país, cantidad de abonados en SMA y telefonía fija, suscriptores de banda ancha fija 
y móvil, cantidad de ancho de banda de Internet por usuario y finalmente se muestra porcentajes de cobertura en cuanto a redes móviles.

El porcentaje de aporte al PIB de cada país por parte de las telecomunicaciones se muestra en la figura 4. Si bien Bolivia tiene un mayor porcentaje, es importante tener en cuenta que el valor del PIB, en dólares, de Bolivia es menor que al de Ecuador.

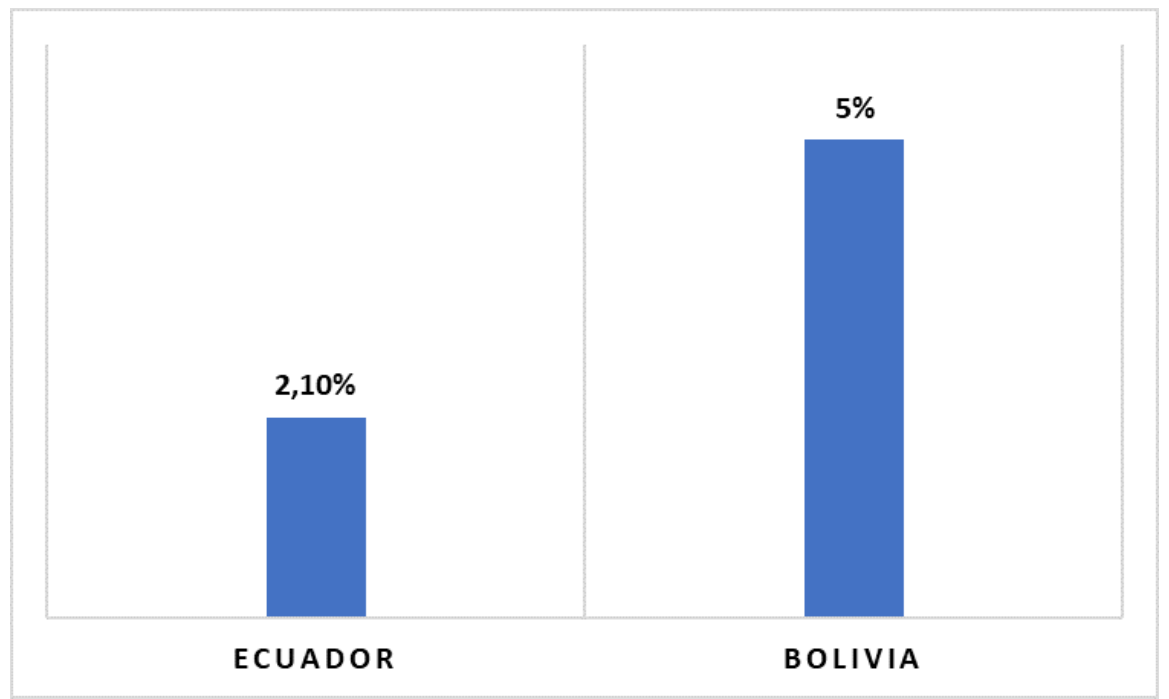

Figura 4. Aporte de las telecomunicaciones al PIB por país.

Debido a que el acceso a los servicios de telecomunicaciones depende directamente de su costo, este representa un indicador clave. Los precios de los planes de servicio de internet fijo representan el mínimo gasto en el que un hogar debe incurrir para tener acceso a este servicio. En el caso de Ecuador la tarifa de un paquete básico de banda ancha fija es de 20.2 dólares mientras que en Bolivia el precio es de 46.6 dólares (Aileen, 2015) como se muestra en la figura 5. Como se puede observar en Bolivia resulta más costoso contratar un plan de banda ancha fija mientras que en Ecuador es más económico.

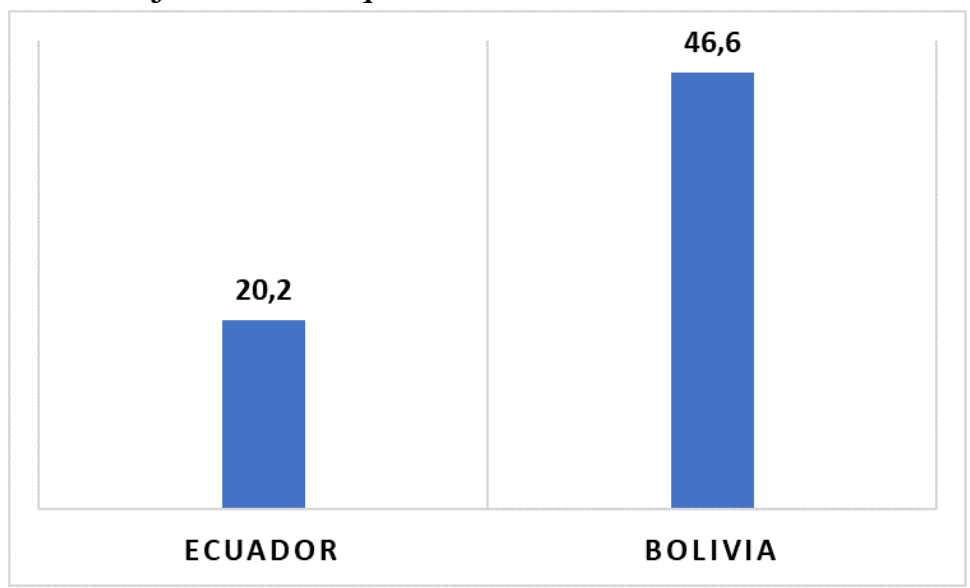

Figura 5. Costo del plan básico de Internet fijo en USD. 
Para este análisis se considera necesario tomar en cuenta el salario básico establecido en la normativa de cada país con el fin de determinar el porcentaje que representa en este la contratación del servicio de Internet fijo. En Ecuador el salario básico es de 386 dólares (INEC, 2018) y en Bolivia es de 298,08 dólares (Paz \& Melendres, 2018). Por lo tanto, en Ecuador contratar un plan básico de Internet fijo representa el 5.23\% del salario mientras que en el caso de Bolivia representa el 15.63\% como se puede observar en la figura 6 .

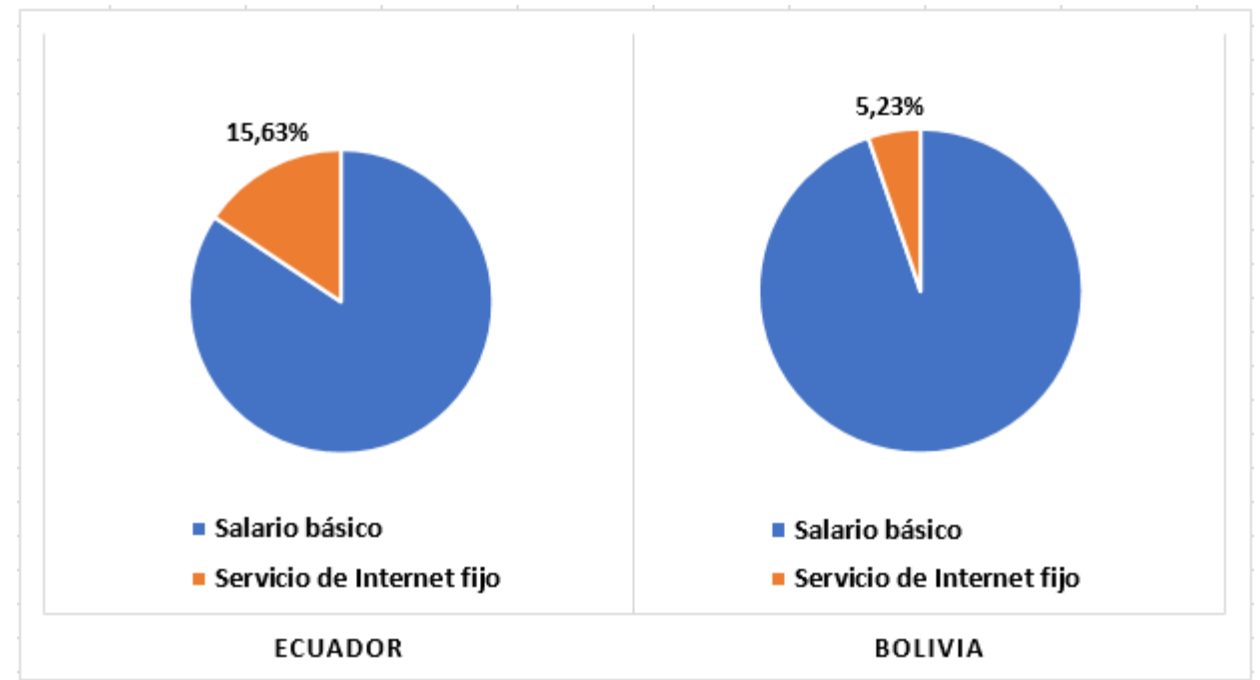

Figura 6. Porcentaje de costo del servicio de Internet fijo con respecto al salario básico.

La cantidad de abonados, tanto en SMA como en telefonía fija, por cada 100 habitantes fija se muestra en la figura 7. En Bolivia se tiene un porcentaje de acogida mayor de SMA, con respecto a Ecuador, mientras que el servicio de telefonía fija muestra mejores valores de aceptación en Ecuador en relación a Bolivia.

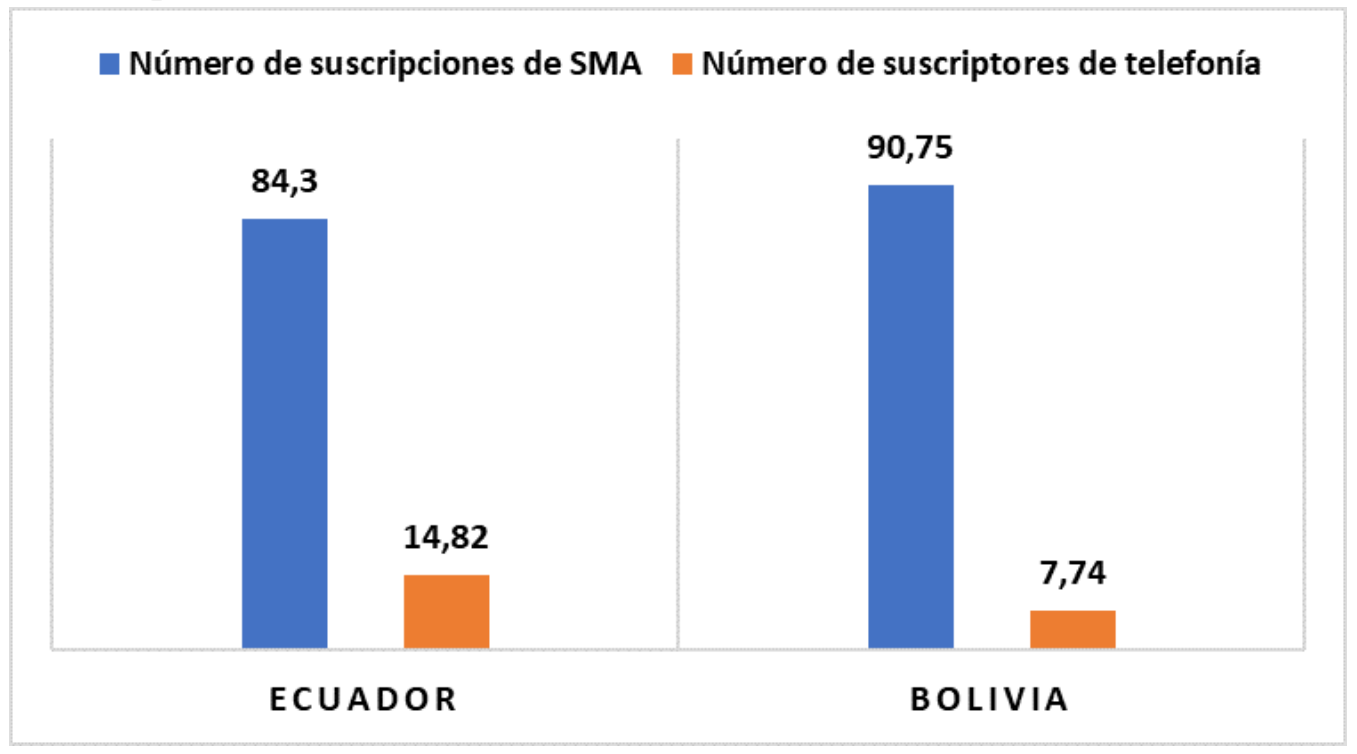

Figura 7. Número de suscripciones del SMA y telefonía fija cada 100 habitantes. Fuente: 
(ITU, 2017b).

Bolivia a diferencia de Ecuador, muestra un mayor porcentaje de suscriptores de banda ancha móvil (figura 8). Con respecto a la cantidad de suscriptores de banda ancha fija se puede notar mejores cifras para Ecuador.

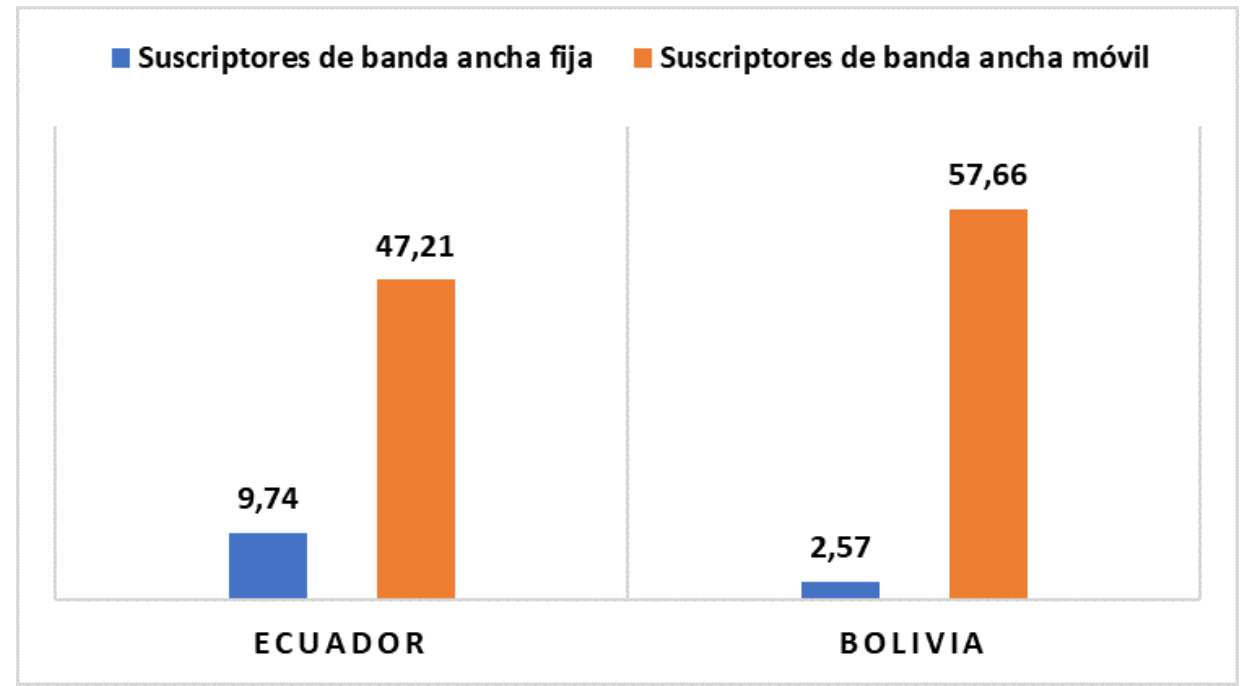

Figura 8. Suscriptores de banda ancha fija y móvil cada 100 habitantes. Fuente: (ITU, 2017b).

En cuestión de ancho de banda de Internet por usuario, Ecuador muestra valores superiores a los de Bolivia. En Ecuador se tiene $7.33 \mathrm{Kbps}$ más de ancho de banda con respecto a Bolivia (figura 9).

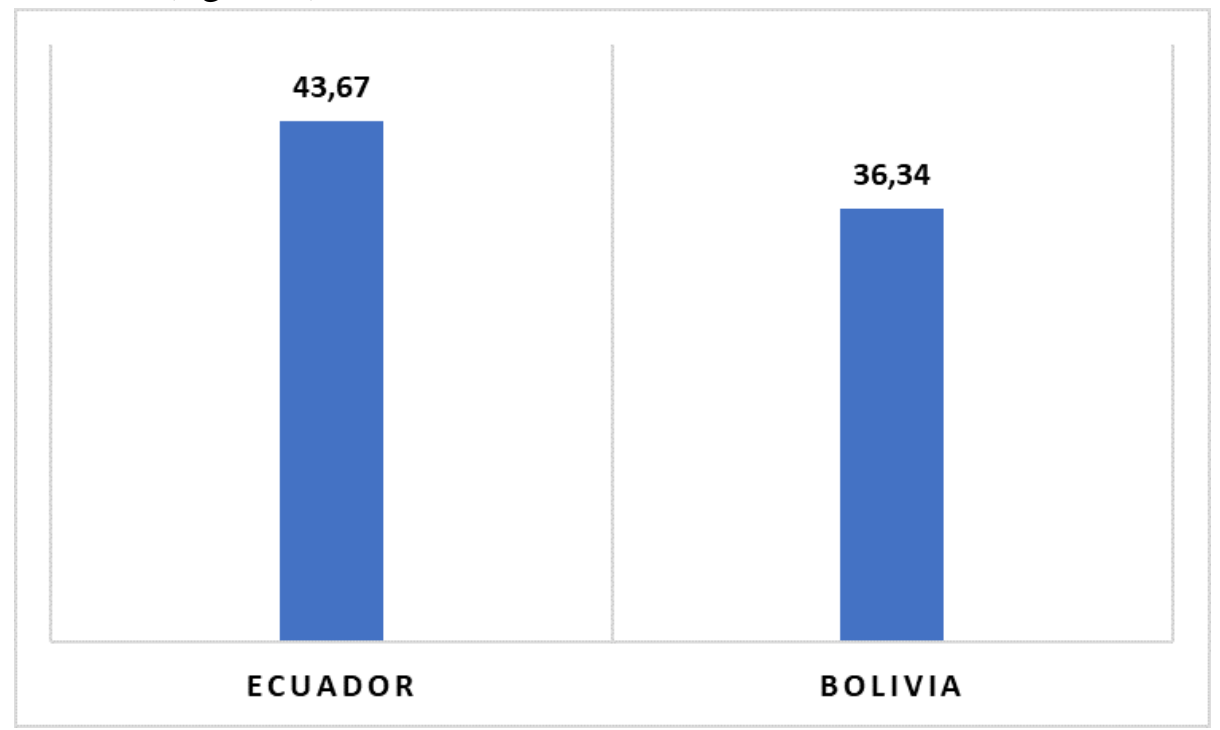

Figura 9. Ancho de banda de internet por usuario en Kbps por país. Fuente: (ITU, 2017b).

Con respecto a cobertura de redes móviles de 3ra y 4ta generación por porcentaje de población se tiene que: en Ecuador, hay un mayor porcentaje de cobertura de redes $3 \mathrm{G}$, 
aproximadamente un $13 \%$ más de cobertura con respecto a Bolivia. En el ámbito de redes 4G Bolivia muestra un 10\% más de cobertura comparando con Ecuador (figura 10).

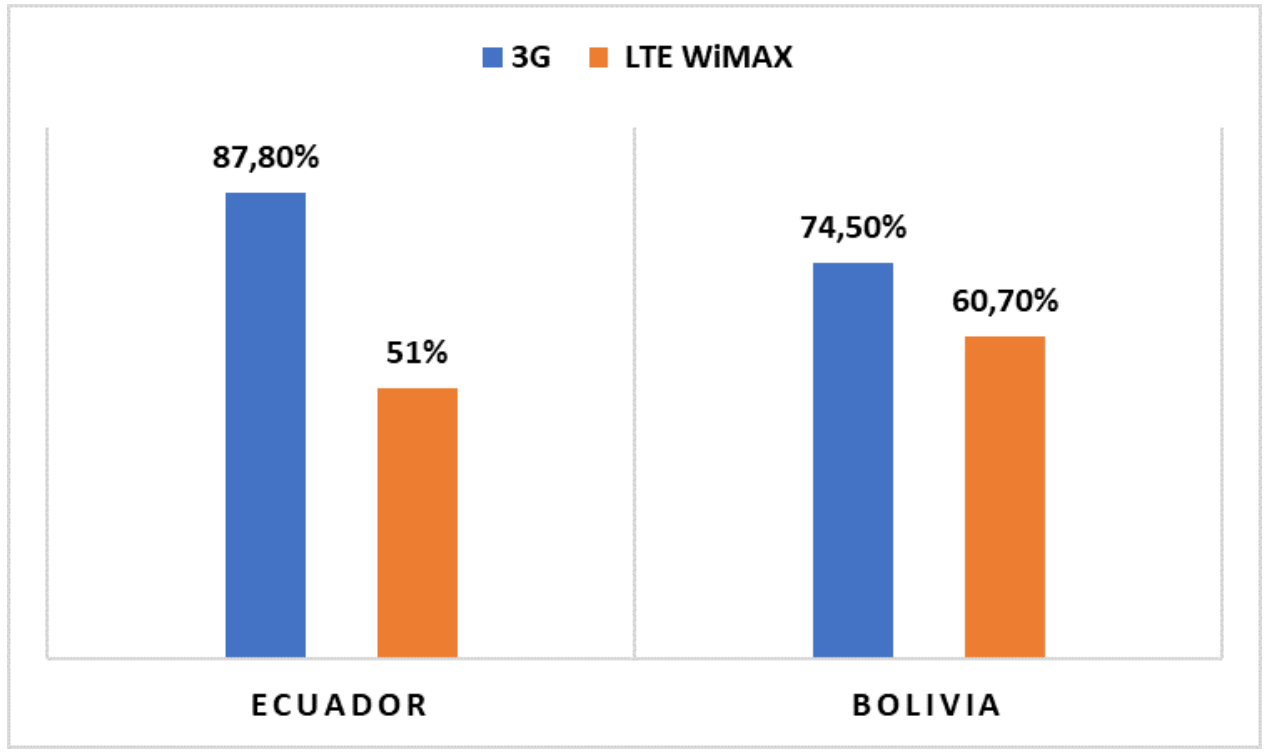

Figura 10. Cobertura 3G y LTE WiMAX por porcentaje de población. Fuente: (ITU, 2017c).

\section{Conclusiones .}

- El sector de las Telecomunicaciones tanto en Ecuador como en Bolivia ha ido en crecimiento. El potencial de los mercados en este sector en ambos países es grande puesto que los porcentajes de cobertura de redes móviles, especialmente de 4ta generación, aún son bajos. Por lo tanto, ante la creciente demanda de servicios que exigen mayores recursos de conexión, existen grandes oportunidades para nuevas empresas que se encuentren interesadas en brindar sus servicios en cualquiera de estos dos países.

- En el ámbito legal tanto Ecuador como Bolivia cuentan con una ley que rige las telecomunicaciones en cada país. Los tiempos de concesión para las operadoras son semejantes, igualmente el porcentaje de espectro radioeléctrico destinado a ese tipo de redes es del $33 \%$ en los dos países. Un factor que los diferencia y pone a Bolivia por debajo de Ecuador es la portabilidad numérica. En Bolivia no existe un reglamento que garantice este servicio a los abonados, y algo más preocupante es que el Estado haya dado paso al pedido de las operadoras de postergar la implementación de dicho servicio. En consecuencia, se puede pensar que en Bolivia existe un oligopolio en el sector de las telecomunicaciones ya que la portabilidad numérica fomenta la competencia y es un factor clave para el ingreso de nuevas operadoras.

- Desde el punto de vista técnico, Bolivia se ve afectado de gran manera por ser un país mediterráneo. Si bien se accede por otros países, como Chile y Argentina, a 
conexiones de cables submarinos estas transiciones de país a país aumentan la latencia, lo cual perjudica directamente a la experiencia del usuario de Internet. Además, en caso de querer desplegar un cable submarino se incurriría en un doble gasto puesto que se debe pagar una concesión tanto en el país de tránsito como en Bolivia. De igual forma los trámites legales se deberían realizar en ambos países. Ecuador al contar con un reglamento para la interoperabilidad numérica y con una posición geográfica más favorable comparado con Bolivia, presenta mejores condiciones tanto técnicas como legales para el despliegue de una nueva red de telecomunicaciones.

\section{Agradecimiento.}

Los autores agradecen el apoyo del Grupo de Investigación Redes Robustas, Sustentables y Seguras SRSNet del Departamento de Ciencias de la Computación y Electrónica de la Universidad Técnica Particular de Loja UTPL.

\section{Referencias bibliográficas.}

\section{ADSIB. (2011). L0164: LEY GENERAL DE TELECOMUNICACIONES,} TECNOLOGIAS DE INFORMACION Y COMUNICACION. Retrieved May 12, 2018, from https://adsib.gob.bo/L0164-LEY-GENERAL-DE

Aileen, A. (2015). Banda ancha en América Latina: Precios y tendencias del mercado.

ARCOTEL. (2016). Resolución 05-03-Arcotel-2016, (593), 1-136. Retrieved from http://www.arcotel.gob.ec/wp-content/uploads/2015/06/RESOLUCIÓN-04-03ARCOTEL-2016-PDF-1.pdf

ARCOTEL. (2018a). Boletín estadístico, 2. Retrieved from http://www.sedmagdalena.gov.co/link_transparencia/2017/Boletin_Estadistico_Gener al_V3.pdf

ARCOTEL. (2018b). Servicio Móvil Avanzado (SMA) | Agencia de Regulación y Control de las Telecomunicaciones | Ecuador. Retrieved May 13, 2018, from http://www.arcotel.gob.ec/servicio-movil-avanzado-sma/

Asamblea Nacional. (2013). Ley Orgánica de Comunicación, 1-24.

Asamblea Nacional. (2015). Ley Orgánica De Telecomunicaciones. Registro Oficial Órgano Del Gobierno Del Ecuador, Tercer Sup, 1-40. Retrieved from http://www.telecomunicaciones.gob.ec/wp-content/uploads/downloads/2016/05/LeyOrgánica-de-Telecomunicaciones.pdf 
ATT. (2012). Reglamento General a la Ley N 164 General de Telecomunicaciones y Tecnologías de Información y Comunicación, para el Sector de Telecomunicaciones. Retrieved May 12, 2018, from https://att.gob.bo/sites/default/files/archivospdf/Anexo al decreto 1391.pdf

ATT. (2016). Memoria institucional 2016 ATT - Bolivia. La Paz. Retrieved from https://issuu.com/attbolivia/docs/memoria_att_2016_final

ATT. (2017a). Estadística Sectorial de Telecomunicaciones y TIC.

ATT. (2017b). Estado de Situación de las Telecomunicaciones en Bolivia. LA Paz.

Cabrera, P., \& Gabarr, P. P. (2017). LA GOBERNANZA DE LAS TELECOMUNICACIONES, 137.

Campero, J. (2016). Infraestructura de telecomunicaciones y TIC en Bolivia. LA Paz. Retrieved from https://internetbolivia.org/wp-content/uploads/2017/05/Camperomerged.pdf

CLARO. (2018). Claro Ecuador. Retrieved May 13, 2018, from https://www.claro.com.ec/personas/

CNT EP. (2018). Home - Corporación Nacional de Telecomunicaciones. Retrieved May 13, 2018, from https://www.cnt.gob.ec/

CONATEL. (2007). Reglamento de Interconexión, 1-15.

Consulado de Bolivia. (2017). El 95\% de las conexiones por internet en Bolivia se da por telefonía móvil. Retrieved May 13, 2018, from https://www.consuladodebolivia.com.ar/2017/01/15/95-las-conexiones-internetbolivia-se-da-telefonia-movil/

EABOLIVIA. (2013). Satélite Túpac Katari. Retrieved May 13, 2018, from https://www.eabolivia.com/satelite-boliviano-tupac-katari.html

EKOS. (2017). Computerworld Ecuador - Especial Ranking TIC 2017 - Edición 303. Retrieved from http://dp.hpublication.com/publication/514fc6bc/mobile/?alt=1

IBM. (2008). Global Location Trends 2008, (October).

IBM. (2017). Global Location Trends 2017.

INE. (2018). INE - Instituto Nacional de Estadística. Retrieved May 12, 2018, from https://www.ine.gob.bo/ 
INEC. (2018). Informe Ejecutivo Canastas Analíticas Abril 2018, 1-2. Retrieved from http://www.ecuadorencifras.gob.ec/documentos/webinec/Inflacion/canastas/Canastas_2018/Abril-2018/1. Informe_Ejecutivo_Canastas_Analiticas_abr_2018.pdf

ITU. (2017a). ITU | 2017 Global ICT Development Index. Retrieved May 13, 2018, from https://www.itu.int/net4/ITU-D/idi/2017/\#idi2017comparison-tab

ITU. (2017b). ITU | 2017 Global ICT Development Index.

ITU. (2017c). Measuring the Information Society Report.

Jaya, O. (2017). DISEÑO, SIMULACIÓN Y ANÁLISIS SOCIOECONÓMICO Y TÉCNICO PARA LA IMPLEMENTACIÓN DE UN ENLACE ÓPTICO SUBMARINO ENTRE EL ECUADOR CONTINENTAL Y EL ARCHIPIÉLAGO DE GALÁPAGOS.

Ministerio de Telecomunicaciones y de la Sociedad de la Información. (2016). Plan Nacional De Telecomunicaciones Y Tecnologías de Información del Ecuador 20162021. Sector de Telecomunicaciones y Tecnologías de La Información, 1, 66.

Retrieved from https://www.telecomunicaciones.gob.ec/wpcontent/uploads/2016/08/Plan-de-Telecomunicaciones-yTI..pdf\%0Ahttps://www.telecomunicaciones.gob.ec/wpcontent/uploads/2016/08/Plan-de-Telecomunicaciones-yTI..pdf\%0Ahttp://www.telecomunicaciones.gob.ec/wp-content

Montenegro, J., \& Sánchez, J. (2016). DISEÑO DE UNA PLATAFORMA PARA LA VERIFICACIÓN DEL CUMPLIMIENTO DE LOS PARÁMETROS DE CALIDAD POR PARTE DE LAS EMPRESAS QUE ATERRIZAN CABLES SUBMARINOS DE FIBRA ÓPTICA EN EL ECUADOR. Retrieved from https://www.dspace.espol.edu.ec/retrieve/97503/D-103460.pdf

Movistar. (2018). Movistar Ecuador |4G LTE, Telefonía Móvil, Internet, Celulares, Recargas - Movistar. Retrieved May 13, 2018, from https://www.movistar.com.ec/

Paz, C., \& Melendres, M. (2018). Alza salarial del 5,5\% al básico y 3\% al mínimo |EL DEBER. Retrieved June 5, 2018, from https://www.eldeber.com.bo/economia/Alzasalarial-del-55-al-basico-y-3-al-minimo-20180426-0004.html

TELECOMReview. (2015). PCCS Cable Consortium to Interconnect Customers to New Subsea Cable System. Retrieved May 13, 2018, from http://www.telecomreviewna.com/index.php?option=com_content\&view=article\&id= $1079 \% 3$ Apccs-cable-consortium-to-interconnect-customers-to-new-subsea-cablesystem\&catid=62\%3Anovember-december-2015\&Itemid $=124$ 
Velasco, J. (2014). TeleGeography publica su mapa anual de cables submarinos. Retrieved May 13, 2018, from https://hipertextual.com/2014/03/mapa-cables-submarinos-2014

Wholesale Services. (n.d.). Ecuador aumenta su capacidad de conexión al mundo a través de la fibra óptica, 31-32. Retrieved from http://www.imaginar.org/docs/A_ecuador_cable.pdf

Zabala, V., \& Adrade, M. (2016). Ranking TIC 2016 by Ekos. Retrieved from https://issuu.com/ekosnegocios/docs/ranking

Zaballos, A. G., \& López-Rivas, R. (2012). Socioeconomic Impact of Broadband in Latin American and Caribbean Socioeconomic Impact of Broadband in Latin American and Caribbean Countries, (November), 22. Retrieved from https://publications.iadb.org/bitstream/handle/11319/5754/Socioeconomic Impact of Broadband in Latin America and Caribbean Countries.pdf?sequence $=1$

Zaballos, A. G., \& Rodríguez, E. I. (2017). Informe anual del Índice de Desarrollo de la Banda Ancha en América Latina y el Caribe, 129. 


\section{Para citar el artículo indexado.}

Cumbicos J., Jiménez L., Luna M., Valdivieso Á. \& Barona L. . (2018). Comparativa del avance en desarrollo en las telecomunicaciones entre ecuador y Bolivia. Revista electrónica Ciencia Digital 2(3), 10-27. Recuperado desde: http://cienciadigital.org/revistacienciadigital2/index.php/CienciaDigital/article/view/134/11 $\underline{9}$

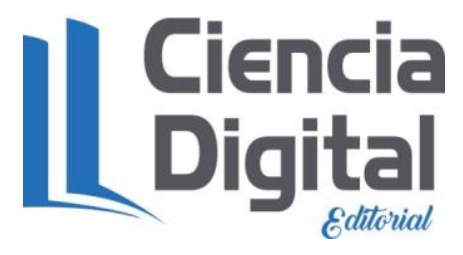

El artículo que se publica es de exclusiva responsabilidad de los autores y no necesariamente reflejan el pensamiento de la Revista Ciencia Digital.

El articulo queda en propiedad de la revista y, por tanto, su publicación parcial y/o total en otro medio tiene que ser autorizado por el director de la Revista Ciencia Digital.
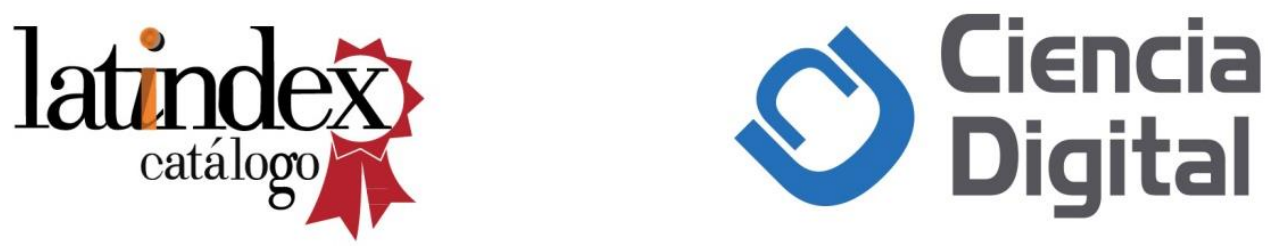